\title{
Global budget for an eruptive active region
}

\section{Equilibrium reconstruction approach}

\author{
A. Bleybel ${ }^{1}$, T. Amari ${ }^{1}$, L. van Driel-Gesztelyi ${ }^{2,3,4,5}$, and K. D. Leka ${ }^{6}$ \\ 1 (CNRS) Centre de Physique Théorique de l'École Polytechnique, 91128 Palaiseau Cedex, France \\ 2 Centre for Plasma Astrophysics, K.U. Leuven, 3001 Leuven, Belgium \\ 3 MSSL, University College London, Holmbury St. Mary, Dorking, Surrey, RH5 6NT, UK \\ ${ }^{4}$ Observatoire de Paris, LESIA, 92195 Meudon, France \\ 5 Konkoly Observatory, 1525 Budapest, Hungary \\ ${ }^{6}$ Colorado Research Associates Division, NorthWest Research Associates, Boulder, CO 80301, USA
}

Received 22 November 2001/ Accepted 28 August 2002

\begin{abstract}
We present results on the magnetic structure of NOAA Active Region \#7912 which was involved in a long duration flare on 14 October 1995, and was the source region for a magnetic cloud observed by the WIND spacecraft from October 18-20. Using vector magnetograms from the Imaging Vector Magnetograph ("IVM"), we reconstruct the magnetic field above this active region, assuming it is in a non-linear force-free state. This reconstruction is used to determine global properties of the active region magnetic field including topology, magnetic energy, and relative magnetic helicity. A comparison of some global quantities before and after the eruptive event is discussed. We show that the magnetic energy and relative helicity of the active region decreased after the eruption, consistent with the ejection of a large amount of helicity (in the magnetic cloud). We also show that the relaxed post-flare state still contains nonlinearities and is not consistent with a linear force-free state as predicted by Taylor's theory of relaxation. These results agree with those of recent numerical simulations concerning plasmoid ejection and helicity redistribution in the disruption of magnetic configurations. We propose as an explanation that the anchoring of field lines in the photosphere prevents a full cascade to the Taylor state, and that a variational formulation in which the action functional would describe this constraint should be derived.
\end{abstract}

Key words. MHD - Sun: corona - Sun:magnetic fields

\section{Introduction}

It is now accepted that phenomena and structures occurring in the solar atmosphere such as sunspots, prominences, or flares are dominated and governed by the magnetic field. Coronal Mass Ejections (CME) represent one of the largest eruptive phenomena. From a theoretical point of view, CMEs represent a way for the Sun to shed helicity, which is generated mainly in the subphotospheric layers and would accumulate incessantly otherwise, by ejecting it into interplanetary space (Low 1994). This phenomenon of helicity redistribution has been quantitatively considered in Amari and Luciani (2000) in the context of global conservation of magnetic helicity. Magnetic helicity is present in the solar atmosphere in the magnetic structures that model prominences or coronal loops (Low 1994; Amari et al. 1999, DeVore and Antiochos 2000). In various MHD models of large-scale eruptive phenomena, magnetic energy

Send offprint requests to: A. Bleybel, e-mail: bleybel@cpht.polytechnique.fr is first stored in either a flux rope (Amari et al. 2000; Linker et al. 2001) or a sheared arcade (Antiochos et al. 1999) and then released, leading to a new equilibrium state after relaxation. According to Taylor's theory of relaxation (Taylor 1986) (successfully applied to reversed field pinch devices, but not in tokamaks, Bhattacharjee et al. 1980), the relaxed state should be a linear force-free state. It is worth noting that this theory has been applied to the solar corona and other astrophysical contexts (Heyvaerts \& Priest 1984). It has been recently found that, in the context of numerical MHD simulations of confined disruptive events, the relaxed state still presents high nonlinearities (Amari \& Luciani 2000).

A different approach is presented in this paper, one which does not rely on numerical simulations. We consider an observed active region that is known to have flared, compute global quantities such as magnetic energy and total helicity before and after the flare, and analyze the consistency between the observed features and in the context of Taylor theory. We focus on the flaring active region NOAA 7912, in which a long 
duration event (LDE) was observed on 14 October 1995. This flare event was linked to a magnetic cloud observed by WIND between 18-20 October. The type of eruptive event observed is different from the one considered in the numerical simulation cited above, since it is probably a non-confined event during which large-scale reorganization seems to have occurred. As already noted, the physics of the event is dominated by the magnetic field; to compute global physical quantities one must have knowledge of the three dimensional coronal magnetic field. Unfortunately, coronal magnetic field measurements are not routine. One way to access to the coronal magnetic field is to reconstruct the magnetic field above this active region using the values measured in the denser and cooler photosphere as the boundary condition. The reconstruction problem and possible relevant computational methods is still an area of research (McClymont 1997; Amari et al. 1997). Existing approaches range from those which use a potential field to a non-linear force free assumption, including the constant- $\alpha$ linear forcefree models. In the case of a highly sheared magnetic configuration associated with strongly localized electric currents and large values of magnetic energy, non-linear force free models may be more relevant, although constant- $\alpha$ models can be useful for local analysis purpose. Presently, several non-linear force-free field extrapolation methods are in use (Sakurai 1981; Mikic et al. 1994; Roumeliotis 1997; Amari et al. 1999).

In this paper we use one of our Cartesian nonlinear force-free codes XTRAPOL (Amari et al. 1997, see also http://www . cpht.polytechnique.fr/cpht/amari) to reconstruct the coronal magnetic configuration in the preeruption and post-eruption states, using boundary values of the magnetic field in the photosphere from IVM vector magnetograms. We discuss the eruptive event's possible connection with coronal mass ejections and magnetic clouds, supported by observational as well as theoretical (modeling) evidence. The nature of the post-eruption relaxed state (linear or non linear force-free) can be addressed by inspecting the computed solution. Although part of the helicity is ejected with the magnetic cloud into the interplanetary medium, we are able to check the original Taylor's conjecture as well as a weaker version of it which states that the remaining helicity fixes the linear postflare state.

The paper is organized as follows: in Sect. 2 we describe the NOAA Active Region \#7912 and its activity on 14 October 1995. In Sect. 3 we discuss the magnetic data and their analysis. In Sect. 4 we describe the method used for reconstruction and the results. In Sect. 5 we discuss the results and conclude.

\section{The Active Region NOAA 7912 and the LDE}

The AR7912, located in the southern hemisphere, had a magnetic orientation opposite to the general Hale's Law (Fig. 1). Such "reversed-polarity" groups are frequently suspected to be formed as the result of a kink instability (Linton et al. 1996), which creates a "knot" in a submerged flux tube due to very strong twist. One characteristic of this instability is that the sign of the twist in the flux tube must be the same sign as the writhe of the flux tube axis. Based on the longterm (four rotations) evolution of the AR, it was proposed by
López Fuentes et al. (2000) that AR7912, having an opposite sign of twist and writhe, was in contrast formed by a helical flux tube which was itself deformed in the convection zone by external forces while ascending.

On 14 October a C1.6 GOES class long-duration event (LDE) started in AR 7912 somewhat after 5:00 UT, reached maximum X-ray flux at 9:21 UT according to the SolarGeophysical Data and lasted for at least 15 hours longer. The GOES curve of the LDE is very spiky (Fig. 2), several flares occurred in the same and neighboring active regions over its duration. The LDE started by loop brightenings in the central part of the AR, during which some of the sigmoid loops became visible (Fig. 3). Loop expansion was already visible at 05:23 UT; by 07:29 UT the expanding loops encountered the magnetic fields of neighboring regions and an "X-point" coronal brightening appeared, presumably the result of inter-activeregion reconnections. The expansion of the AR 7912 continued to where by $08: 22$ (and certainly by $08: 55 \mathrm{UT}$ ), the span of the fading loops, in projection, became comparable to the solar radius.

A magnetic cloud was observed with the Solar Wind Experiment and the Magnetic Field Instrument on board the WIND spacecraft at 1 AU from 18-20 October 1995. Magnetic modeling of the observations showed that the cloud could be well approximated with a flux rope of right-handed twist (positive helicity; Lepping et al. 1997). It was proposed by van Driel-Gesztelyi et al. (2000) that the magnetic cloud was launched from AR 7912: the positive (right-handed) twist of the cloud agreed with the sign of the twist in the expanding coronal loops as indicated by their forward S-shape. We will show below that the magnetic helicity in the AR indeed had a dominantly positive sign. This positive twist corresponds to the hemispheric helicity pattern found by Pevtsov et al. (1995), who showed that the majority (nearly $70 \%$ ) of active regions on the south (north) hemisphere has positive (negative) helicity.

\section{Photospheric magnetic field}

The magnetic field is most readily measured in the photosphere using the Zeeman effect for magnetically-sensitive solar spectral lines. The current lack of direct measures of the coronal magnetic fields above active regions necessitates the use of modeling approaches to reconstruct the coronal fields. To reconstruct the magnetic field in the corona $(\{z>0\}$, where $z$ is the vertical coordinate), one needs the photospheric field to be well determined.

Photospheric vector magnetograms from the Imaging Vector Magnetograph ("IVM"; Mickey et al. 1996; Labonte et al. 1999) were used to perform our calculations. The magnetograms were taken at Mees Solar Observatory on Haleakalā, on 14 October 1995, at 00:20 UT and 17:55 UT. The capabilities of the IVM encompass those of pre-existing magnetographs, with a time resolution of $10 \mathrm{~min}$ and an effective pixel size of 1.1 arcsec. There is one caution to note with this type of magnetograph: IVM may make scale errors in the magnetic flux strength, but accurately represents the spatial structure. This is in contrast to spectrometer-based designs such as HSP which may generate more accurate magnetic flux values but 


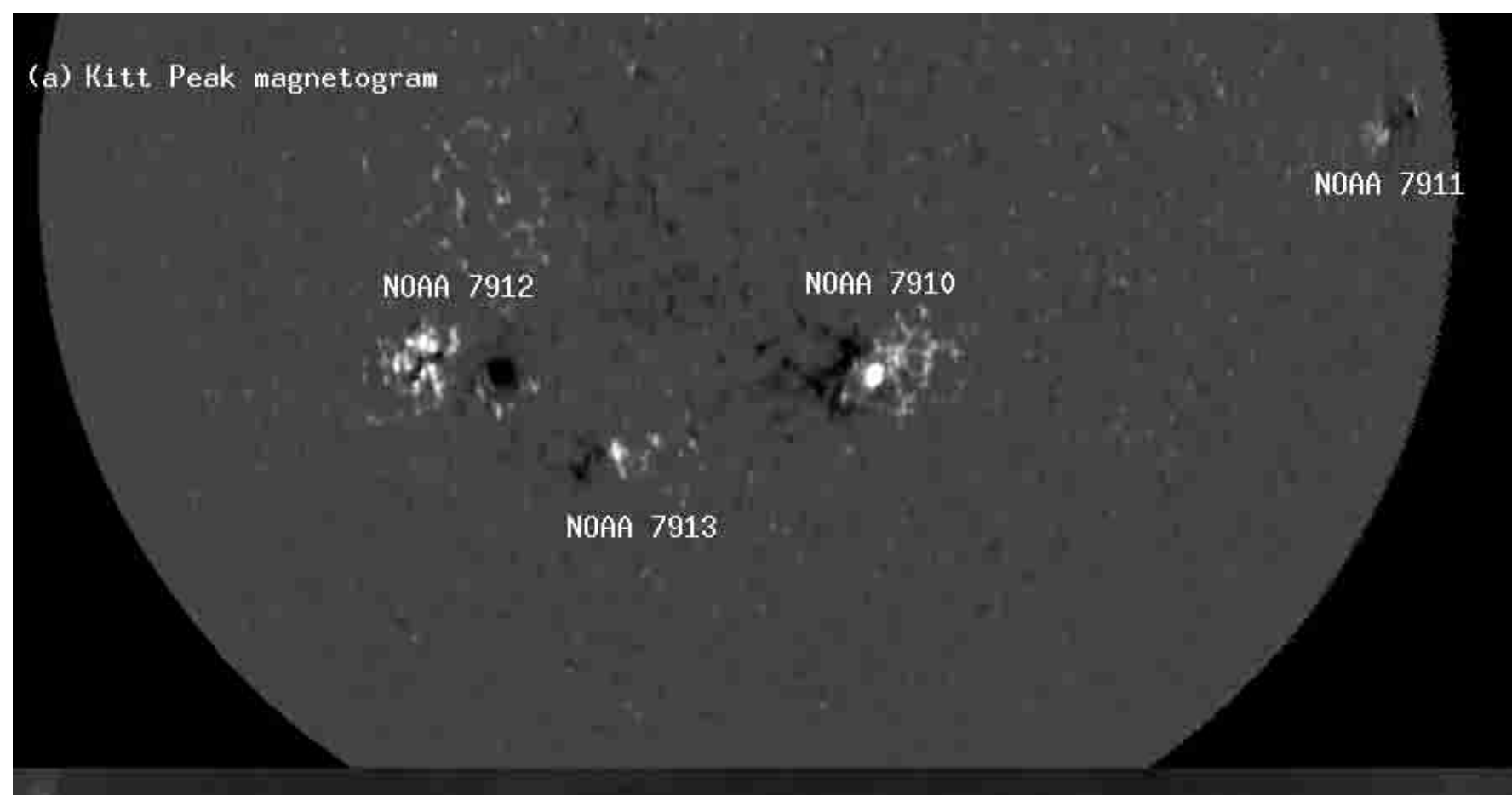

(b) Yohkoh/SXT image

Fig. 1. a) Kitt Peak magnetic map and b) Yohkoh/SXT soft X-ray observation on 14 Oct. 1995. Note the S-shaped loops in the reversed polarity active region NOAA 7912. The twisted loops are in expansion in the X-ray image, which was taken before the peak of the LDE at 07:29 UT (Figure taken from van Driel-Gesztelyi et al. 2000).

introduce spatial distortions. The magnetic flux vector $\boldsymbol{B}$ was derived using the weak field approximation (Jefferies et al. 1989) applied to the observed Stokes polarization spectra (Fig. 4).

The transverse field components were corrected for the $180^{\circ}$ ambiguity using an iterative method (Canfield et al. 1993). In order to reconstruct the coronal magnetic field, the observed photospheric field is then transformed to heliospheric coordinates, i.e. the coordinates in the frame of reference attached to the Sun, with the $z$ axis normal to the photosphere.

The electric current $J_{z}$ is calculated from Ampere's law using $B_{x}$ and $B_{y}$ values on the photosphere.

The values of $\alpha$ in the photosphere are calculated using the photospheric magnetic field $B_{z}$ and the electric current $J_{z}$, giving $\alpha=4 \pi J_{z} / B_{z}$, in cgs units.

Our non-dimensional units are defined such that the fieldof-view of an IVM magnetogram is 1, and photospheric values 


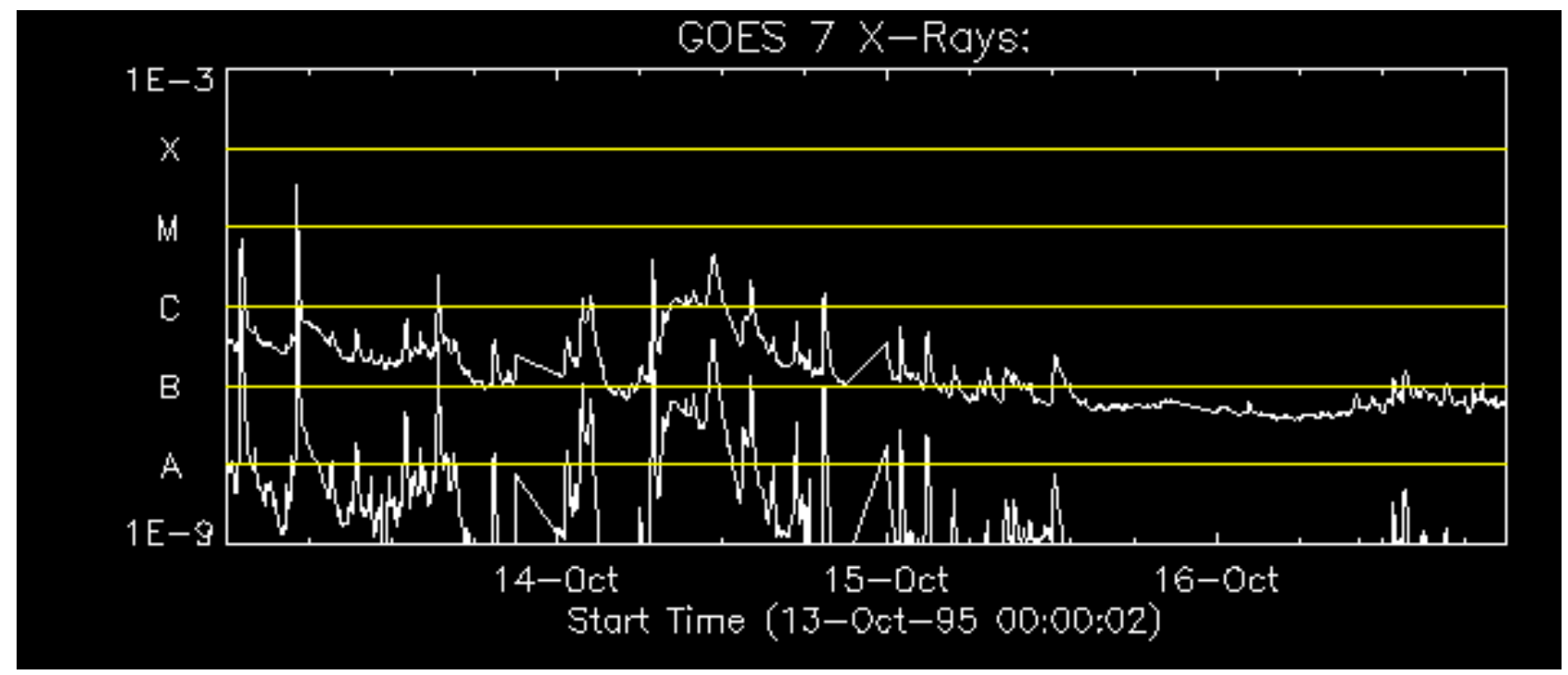

Fig. 2. Integrated solar soft X-ray emission in the 1-8 A range (GOES-7) between 13-16 October 1995. Note the long-duration event (LDE) between 5-24 UT on 14 October which was related to the launch of the 18-20 October 1995 magnetic cloud observed with WIND.

of $B$ are scaled with the maximum values for $B_{z}$ set to 1 . For instance, the maximum of $B_{z}$ was found to be $2430 \mathrm{G}$, and the length of the initial IVM magnetograms is $L_{0}=214 \mathrm{Mm}$. This choice implies the values of $\alpha$ in those units shown in Figs. 6, 7. The factor of conversion (used to transform $\alpha$ from cgs units to our units) used in our calculation was found to be $2689.200=$ $4 \pi \times 10^{-6} L_{0}$. Also, the mean value of $\alpha$ in the active region was found to be $(77 \pm 0.14) E-4 \mathrm{Mm}^{-1}$ before the eruption and $(167.5 \pm 0.38) E-4 \mathrm{Mm}^{-1}$ after the eruption. Note that mean values of $\alpha$ in the main spot were $(176.3 \pm 0.32) E-4 \mathrm{Mm}^{-1}$ before the eruption and $(224.0 \pm 0.51) E-4 \mathrm{Mm}^{-1}$ after the eruption. In the weak field region we found a larger noise level due to the noise in $B_{x}$ and $B_{y}$ and so we introduce a cut-off of 200 Gauss on these components. Note that introducing the cut-off on these components was done before making the transformation to heliospheric coordinates, because transverse components are more subject to noise than line-of-sight component (in the observer's frame). We have tried several cut-offs (50,100 and 150 Gauss) on $B_{z}$ and we have chosen a cut-off of 150 Gauss to prevent a large $\alpha$ that would arise from the weak normal photospheric magnetic field values. This cut-off is only used for computing $\alpha$ while $B_{z}$ used as a boundary condition is the exact $B_{z}$ obtained from measurements. The distribution of $\alpha$ shows already that $\alpha$ is not uniform, even on small scales (Fig. 5). Note that changes of sign of $\alpha$ in the main spot may be related to the presence of return currents, and that both signs of $\alpha$ exist in the following part of the region. These two regions (the main spot and the following part) correspond to the same distribution in the map of $B_{z}$, and $\alpha$ has the highest values there. Some noise in $\alpha$ is present in the region of weak $B_{z}$, that is close to the edges of the magnetogram. To summarize results from $2 \mathrm{D}$ data analysis, we found that:

- $\alpha$ is not constant even on small scales i.e. there are positive and negative values of $\alpha$ in neighboring regions.

- The dominant sign of $\alpha$ is positive in AR7912 (as can be seen from the mean values of $\alpha$ ).
These results indicate that a main sign of the twist may exist, as emphasized by Pevtsov et al. (1995, 1997). Finally, Fig. 6 and 7 show histograms of $\alpha$ in the active region both before and after the eruption, and the histograms show clearly that $\alpha$ is not constant in absolute value and sign in the active region before or after the eruption. One would expect (Sect. 5) that after the relaxation, $\alpha$ should become constant, according to Taylor's theory. This will be discussed later in this paper.

\section{Reconstruction of the coronal magnetic field}

The problem of magnetic field extrapolation has been given a lot of attention during recent years. Attempts to deduce the magnetic field from photospheric boundary data can fail, in part due to numerical and technical problems: for example the vertical integration method (hereafter VIM) (see Amari \& Démoulin 1992; Démoulin et al. 1992; McClymont 1997; Amari, Boulmezaoud \& Maday 1999) suffers from exponentially growing errors with height $z$ associated with the mathematically ill-posed nature of problem.

The usual procedure to perform this study now consists of modeling a constant- $\alpha$ field (or even sometime a potential field - with $\alpha=0$ ), and tries to match the observed coronal structures, thus deducing a posteriori the value of $\alpha$ ).

Because of the strong non-uniformity of $\alpha$ shown at the photospheric level, we choose to assume that the corona is in a non-linear force-free (and therefore non-constant- $\alpha$ ) state (Amari et al. 1997, 1999).

\subsection{Formulation of the problem}

The corona is assumed to be described by the equilibrium of magnetic field in the domain $\Omega=\{z>0\}$, where the plasma pressure and gravitational forces are neglected. The equations of this problem are the force-free equations (Parker 1979):

$\nabla \times \boldsymbol{B}=\alpha(\boldsymbol{r}) \boldsymbol{B} \quad$ in $\Omega$

$\nabla . \boldsymbol{B}=0$, 


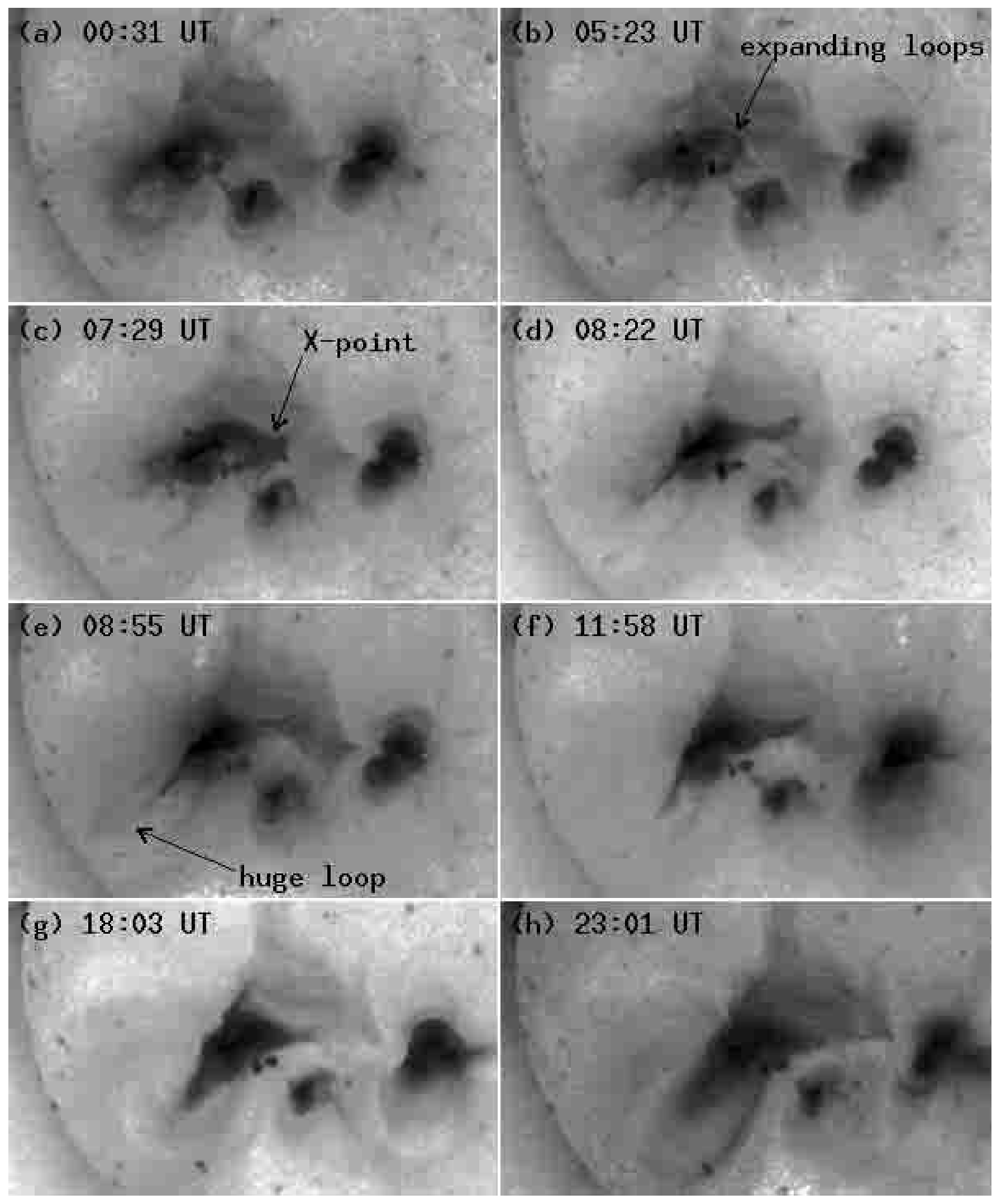

Fig. 3. Evolution of the soft X-ray structures on 14 October 1995 as observed with Yohkoh/SXT. The flare starts with brightenings of short loops in the center of the AR. Expansion of longer external loops begins after 05 UT. By 07:29 UT the expanding loops encounter the magnetic fields of neighboring regions and an X-point forms, indicating inter-AR reconnections. The continuing expansion of AR 7912 loops are seen at 08:22 and especially at 08:55 UT, when a dim "huge" loop can be seen. The expansion of the coronal loops is related to the launch of the magnetic cloud (figure courtesy van Driel-Gesztelyi et al. 2000). 
in which $\alpha(\boldsymbol{r})$ as well as $\boldsymbol{B}$ are unknowns. The boundary conditions which are required for this mixed elliptic-hyperbolic problem are:

$\left.B_{n}\right|_{\partial \Omega}=b_{0}$

for the elliptic part, where $b_{0}$ is some regular function, and

$\left.\alpha\right|_{\partial \Omega^{+}}=\alpha_{0}$

for the hyperbolic part of the problem with $\alpha_{0}$ a bounded function and $\partial \Omega^{+}$the part of $\partial \Omega$ on which $B_{z}>0$.

\subsection{The reconstruction scheme}

One then defines a sequence $\left(\alpha_{0_{p}}\right)_{1 \leq p \leq P}$ for which each term corresponds to a boundary value problems for $\boldsymbol{A}_{p}$. This procedure represents the progressive injection of $\alpha$ at the boundary, and improves the convergence of the classical Grad-Rubin scheme (Grad \& Rubin 1958). The computational domain $\Omega$ is defined such that instead of being unbounded as in the theoretical problem, it is supposed to be a bounded cubic box and discretized as $\Omega_{h}$ using a staggered non-uniform mesh. On the lateral and top boundaries of the box, we set $\boldsymbol{B}_{\mathrm{n}}=0$, with the following boundary conditions for $\boldsymbol{A}$, owing to the "Boundary Flux Gauge" (Amari et al. 1997):

$\nabla \cdot \boldsymbol{A}=0$, in $\Omega$

$\nabla_{\mathrm{t}} \boldsymbol{A}_{\mathrm{t}}=0$, on $\partial \Omega$

$\partial_{\mathrm{n}} \cdot \boldsymbol{A}_{\mathrm{n}}=0$ on $\partial \Omega$

where the subscripts "t" and " $\mathrm{n}$ " stand for the tangential and normal components with respect to the boundary $\partial \Omega$.

The hyperbolic part of the BVP associated to $\alpha$ is solved using a characteristics method approach. As the value of $\alpha$ is constant along a field line, one goes backward along the characteristics or forward if the photospheric values of $\alpha$ on $\partial \Omega^{-}$ are smoother. During the process of computing $\alpha$ one can interpolate $\alpha$ from its nearest neighbors already computed in the domain $\Omega$. The existence of solutions (Boulmezaoud \& Amari 2000) being ensured, the numerical procedure used to solve BVP (1)-(4) is explained in detail in Amari et al. (1996-1999).

We used a non-uniform mesh in the three directions and performed computation at two different resolutions $(n x=92$, $n y=52, n z=39)$ and $(n x=148, n y=88, n z=70)$, leading to the same quantitative results. The non-uniform mesh is concentrated on the leading spot and the mixed polarity region. To mimic the boundary conditions of weak remote field, we add some points in each directions in which the field vanishes, and smooth the data in the transition region. Note that in general this might not be exact since there may exist an overlaying confining large-scale magnetic field. This can actually be taken into account by completing the longitudinal photospheric magnetogram with full disk data, if those data are available. Full flux-balance is an important condition for a correct reconstruction. Our preliminary study of this active region has shown that partial coverage of a full active region, such as with HSP data

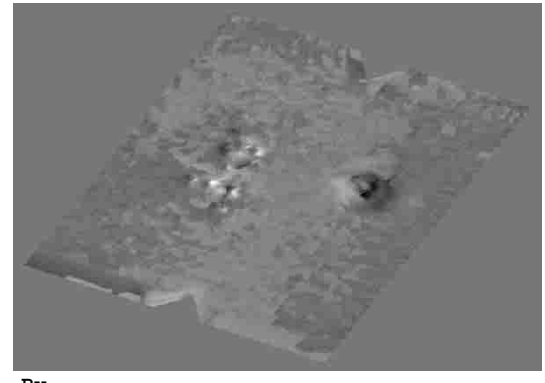

$\mathrm{Bx}$

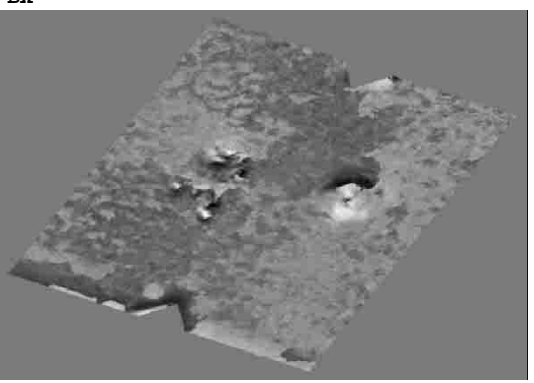

By
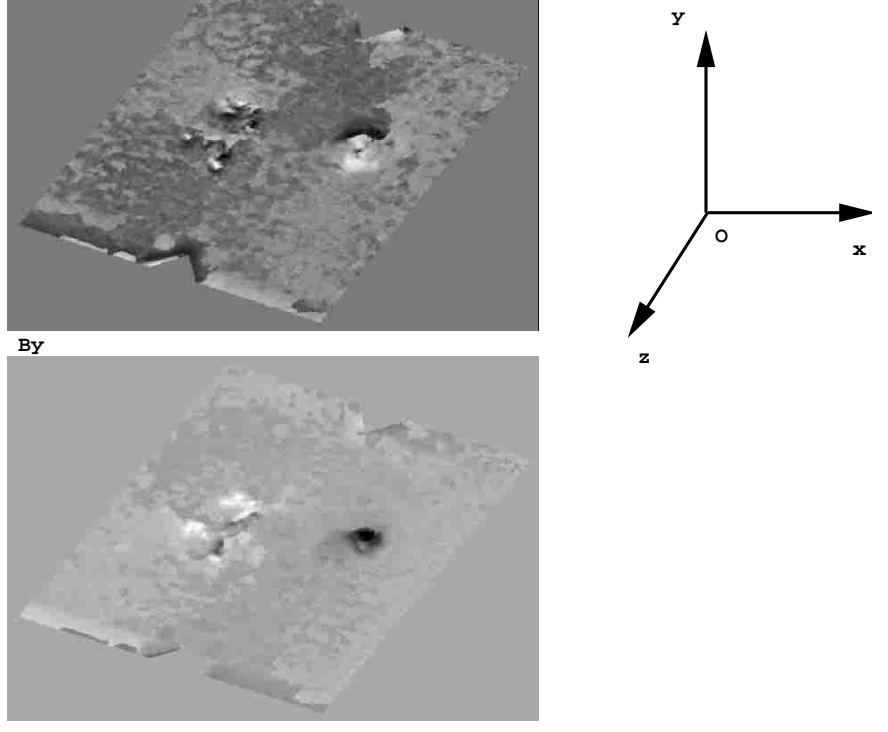

$\mathrm{Bz}$

Fig. 4. The three components of the magnetic field, $B_{x}, B_{y}$ and $B_{z}$ respectively from top to bottom, measured with the IVM (light: positive, dark: negative), with a cutoff on the transverse field of $200 \mathrm{G}$ to avoid large spurious current resulting from noisy data. The axes shown correspond to the heliospheric frame of coordinates.

from 14 Oct., can lead to erroneous results as the computed magnetic structure is not arbitrarily closed. There is one other important point to make before we proceed. The method used relies on the assumption of exact magnetic equilibrium. It follows that the iterative procedure does not reach a solution of the numerical problem in the case of a dynamical state (even slow) or a configuration far from equilibrium. This might be related to the difficulty encountered when reaching finite large values of $\alpha$ : there is no uniqueness theorem for arbitrary values of $\alpha$ even in a bounded domain. A recent general existence theorem (Boulmezaoud \& Amari 2000) has been proved, while uniqueness is only proved (Bineau 1972) for solutions in a functional space excluding $X$ points and current sheets and for small values of $\alpha$. Furthermore, existence of a solution is proved for a bounded domain, while although bounded, our problem actually mimics the open unbounded half space boundary value problem. We then also know that existence is not always ensured since there are non-existence theorems (Aly 1984). One other important source of problems is the method used for solving the $180^{\circ}$ ambiguity in the transverse magnetic field, which may introduce severe errors, e.g.in the topology of the computed configurations. For example, using the potential 


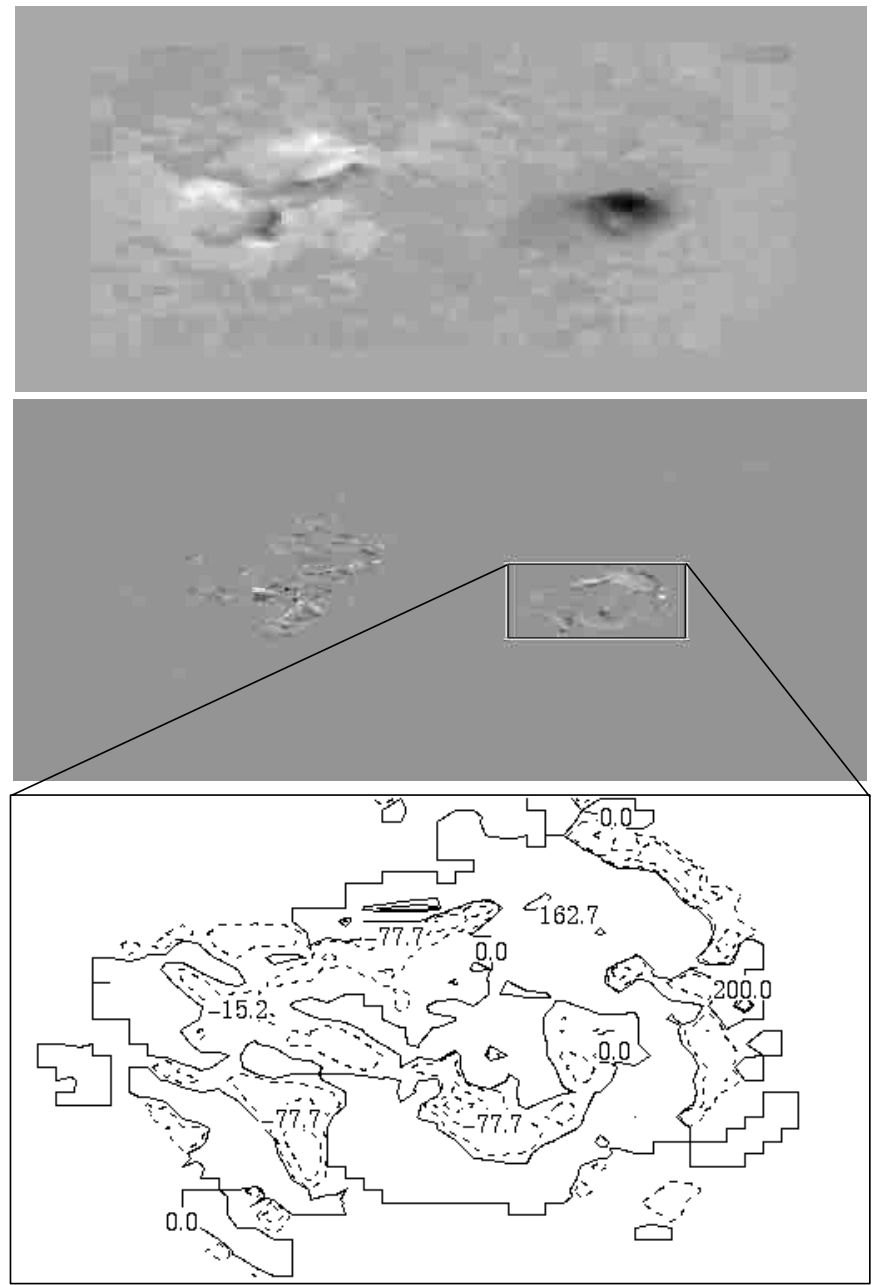

Fig. 5. Map of $B_{z}$ (top), $\alpha$ (center) (light: positive, dark: negative) and contour plot of values of $\alpha$ in the main spot (bottom). Note changes in $\alpha$ even on small scales.

magnetic field as a reference field may lead to erroneous estimations of relative helicity in the case of highly sheared magnetic configurations and would even fail to reproduce a twisted configuration containing a flux rope in equilibrium.

Therefore we generally decide to stop the iterative procedure before reaching the critical phase of the computation. The practical criteria used to determine the correctness of the computation are: i) the convergence of the iterative procedure, ii) the relaxation of the computed state to a weak value of the Lorentz force and iii) a constant value for the magnetic energy with no further oscillations.

\subsection{Results}

a) Reconstruction of the pre-eruptive state:

Once the solution has been computed, the field lines of the configuration are compared to soft X-ray coronal loops. We found that coronal field lines reproduce the soft X-ray sigmoid as shown in Fig. 8 (top) with the co-aligned images.

There exists a twisted flux tube matching the " $\mathrm{S}$ " shaped SXT loop related to the location of enhanced heating (Fig. 8).

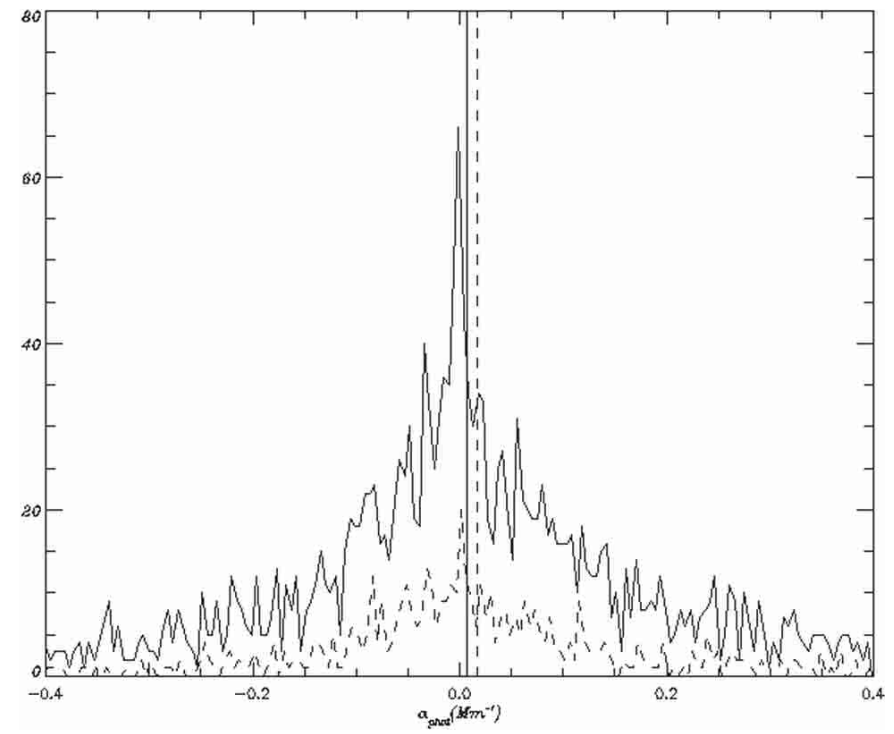

Fig. 6. Histogram showing the distribution of $\alpha$ in the active region NOAA 7912 before the eruption (solid line) and in the main spot of the active region (dashed line). Mean values of $\alpha$ in the region and the main spot are also indicated by vertical lines in the corresponding linestyles.

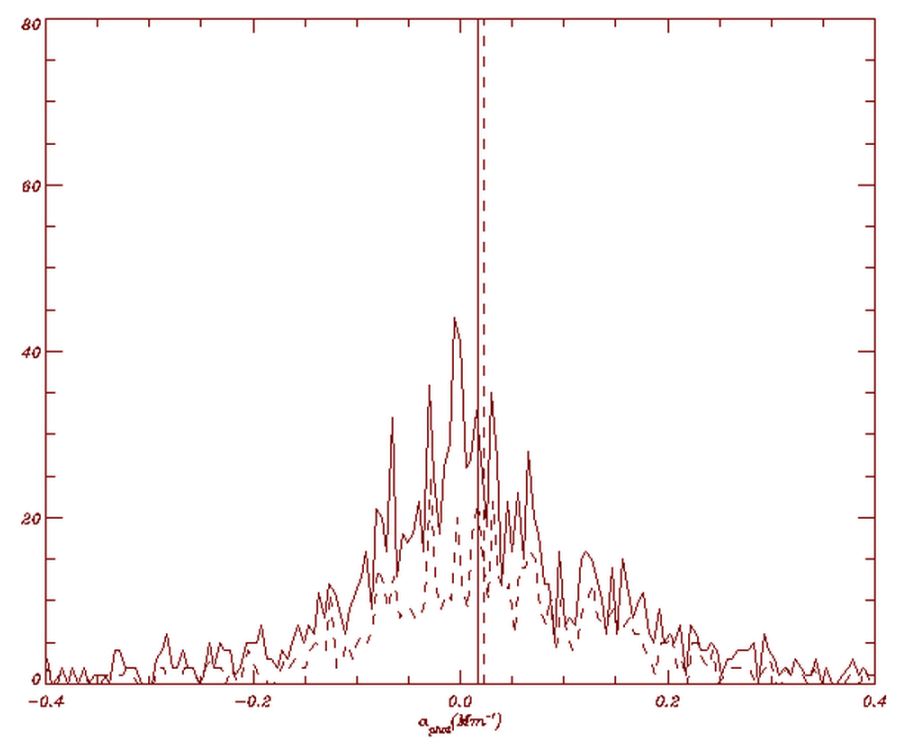

Fig. 7. Histogram showing the distribution of $\alpha$ in the active region NOAA 7912 after the eruption (solid line) and in the main spot of the active region (dashed line). Mean values of $\alpha$ in the region and the main spot are also indicated by vertical lines in the corresponding linestyles.

This flux tube has its footpoints located in a region of negative $\alpha$ (mean value of $\alpha$ is $-0.08395 \mathrm{Mm}^{-1}$ ). We note that field lines make approximately one turn about the axis between the two ends of the flux tube. Because of the large scale of this flux rope, a twist of about $2 \pi$ may be considered as large enough to be close to disruption (Amari \& Aly 1996; for unconfined disruption or Raadu 1972, Hood \& Priest 1979, Amari \& Luciani 1999, Baty 2001 (and refrences therein); for confined disruptions). 
Table 1. Values of different global physical parameters before and after the flare.

\begin{tabular}{lcc}
\hline \hline $\begin{array}{l}\text { Type of data } \\
\text { IVM }\end{array}$ & pre-eruptive & post-eruptive \\
\hline $\begin{array}{l}\text { Magnetic energy } \\
\text { (erg) }\end{array}$ & $(8.41 \pm 0.98) E+32$ & $(5.76 \pm 1.15) E+32$ \\
$\begin{array}{l}\text { Relative helicity } \\
\left(\mathrm{cm}^{4} \mathrm{G}^{2}\right)\end{array}$ & $(1.32 \pm 0.16) E+42$ & $(6.54 \pm 1.25) E+41$ \\
$\begin{array}{l}\text { Corresponding } \\
\alpha\left(\mathrm{Mm}^{-1}\right)\end{array}$ & $(2.58 \pm 0.005) E-2$ & $(1.96 \pm 0.005) E-2$ \\
$\begin{array}{l}\text { Total flux } \\
\left(\mathrm{cm}^{2} \mathrm{G}\right)\end{array}$ & $(8.41 \pm 0.27) E+25$ & $(7.28 \pm 0.19) E+25$ \\
\hline
\end{tabular}

One may notice that there exists another S-shaped, sheared flux tube located at a higher level in the corona which corresponds to a positive mean value of $\alpha\left(0.02845 \mathrm{Mm}^{-1}\right)$. The fact that the two main flux tubes in the active region have $\alpha$ of opposite signs supports the idea that a non-linear force-free field reconstruction is suited for the study of the global configuration involving interaction of flux ropes having counter helicities, rather than linear or potential field reconstructions. Note that similar features were also observed in Mandrini et al. (2000) where two regions of opposite constant $\alpha$ were interacting.

We compute the relative helicity for the non-linear forcefree field calculated above

$\Delta H=\int_{\Omega}\left(A-A_{0}\right) \cdot\left(B+B_{0}\right) \mathrm{d}^{3} r+\int_{\partial \Omega} \kappa\left(B+B_{0}\right) \mathrm{d} s$

as in (Berger \& Field 1984; Finn \& Antonsen 1984; Amari \& Luciani 1999). The second additional surface term in RHS of Eq. (8), (where $\kappa$ is a rather complicated term) arises from the other boundaries of the computational box and vanishes exactly in the case of a magnetic field having no normal component to the boundary. This term is otherwise small when the boundaries are put far away enough in very weak field region. The formula used for calculating the energy is:

$W=\frac{1}{8 \pi} \int B^{2} \mathrm{~d}^{3} V$.

One recalls that $\alpha$ is injected into the configuration in 20 uniform steps. The three criteria described in the previous subsection are applied to consider the degree of validity of the solution along the iterative procedure. We find that along the computational procedure the relative magnetic helicity decreases until iteration 18, and then begins to increase until it reaches a value $50 \%$ higher, while magnetic energy remains nearly constant. Although comparison of field lines produced at the 20th iteration could show at some locations better agreement with the Yohkoh SXT image, we decided to consider the computation valid only up to iteration 18 , characterizing this state as being the closest equilibrium description of the active region state, according to our criteria defined at the end of Sect. 4.2.

The linear force-free field having the same helicity is also computed. The two fields are strongly different as shown in Fig. 8 (top-center). This method for determining the constant$\alpha$ FF field is more meaningful than matching coronal X-ray loops since these loops should be used only for comparison afterward, and since there are various length scales associated with the non-uniformity of $\alpha$. One value of $\alpha$ may account for one X-ray loop, but not for other shorter or longer structures, associated with regions of different $\alpha$. However a constant- $\alpha$ computation, which is much faster (in terms of computational resources) may be useful for focusing on one particular structure, and several such computations might represent a good guess for starting a non-linear computation since such a linear computation does not require transverse field data, which may be very bad for some vector magnetograms. This will be investigated in a forthcoming paper.

b) Reconstruction of the post-eruptive state:

Following the same procedure as for the pre-eruptive state, we compute the state after the eruption, and compare the values calculated with those before the eruption. The magnetic configuration appears to have relaxed after the eruption, as is clearly seen from Yohkoh image (Fig. 9), where the S-shaped loop has nearly disappeared, leaving two interconnected loops. This conjecture is further supported by the smaller values for both the magnetic helicity and energy. As for the preeruptive state, we managed to reproduce the main loops observed in SXT. One of them is a twisted flux tube having positive mean $\alpha\left(=0.03076 \mathrm{Mm}^{-1}\right)$. The other loop consists of two sheared flux tubes, having negative $\left(-0.2292 \mathrm{Mm}^{-1}\right)$ and positive $\left(0.084 \mathrm{Mm}^{-1}\right)$ mean $\alpha$ respectively. One may note that unlike the pre-eruptive flare, the computation was completed up to iteration 20. This might be consistent with the fact that the pre-eruptive state was close to erupt (non equilibrium), with a higher energy.

c) Computational and observational errors:

Estimation of errors shown in Table 1 in the computation of the magnetic energy is made using the same method as in (Klimchuk et al. 1992). This method is based on the virial theorem (in the case of domain $\Omega=\{z \geq 0\}$ ). The contribution of the lateral and top boundaries of the cube of computation are to be found small. We then estimate the observational contribution to relative error in the energy using a discretization of the virial equation, and the same cutoffs defined in Sect. 3 on $B_{z}$ and on $B_{x}$ and $B_{y}$ for the calculation of the error on $W$. Remember that we have already performed these cutoff for the computation of $\alpha$ on the boundary. Since there is no similar expression for the relative helicity, the errors are estimated directly on the volume integral. The errors on the total flux are easily computed from errors on $B_{z}$ and $J_{z}$. For $\alpha$ we computed the root mean square deviation $(\sigma)$ after computing the errors on individual pixels. The other component of the error (i.e. error due to the reconstruction procedure) has been already studied and quantified in Boulmezaoud \& Amari (2000). They found it to be of the order of $2 \%$ in the case of a well-resolved mesh cube, as is our case.

d) Discussion on errors:

Relative errors on magnetic energy were found to be $11.84 \%$ for the pre-eruptive state and $19.0 \%$ for the post-eruptive state, and about the same for magnetic helicity. We can then deduce that these errors do not weaken our conclusions about the loss of magnetic energy and helicity in the active region. It is worth noticing that an estimation of errors due to the $180^{\circ}$ ambiguity removal method used was not done, but may be an important 

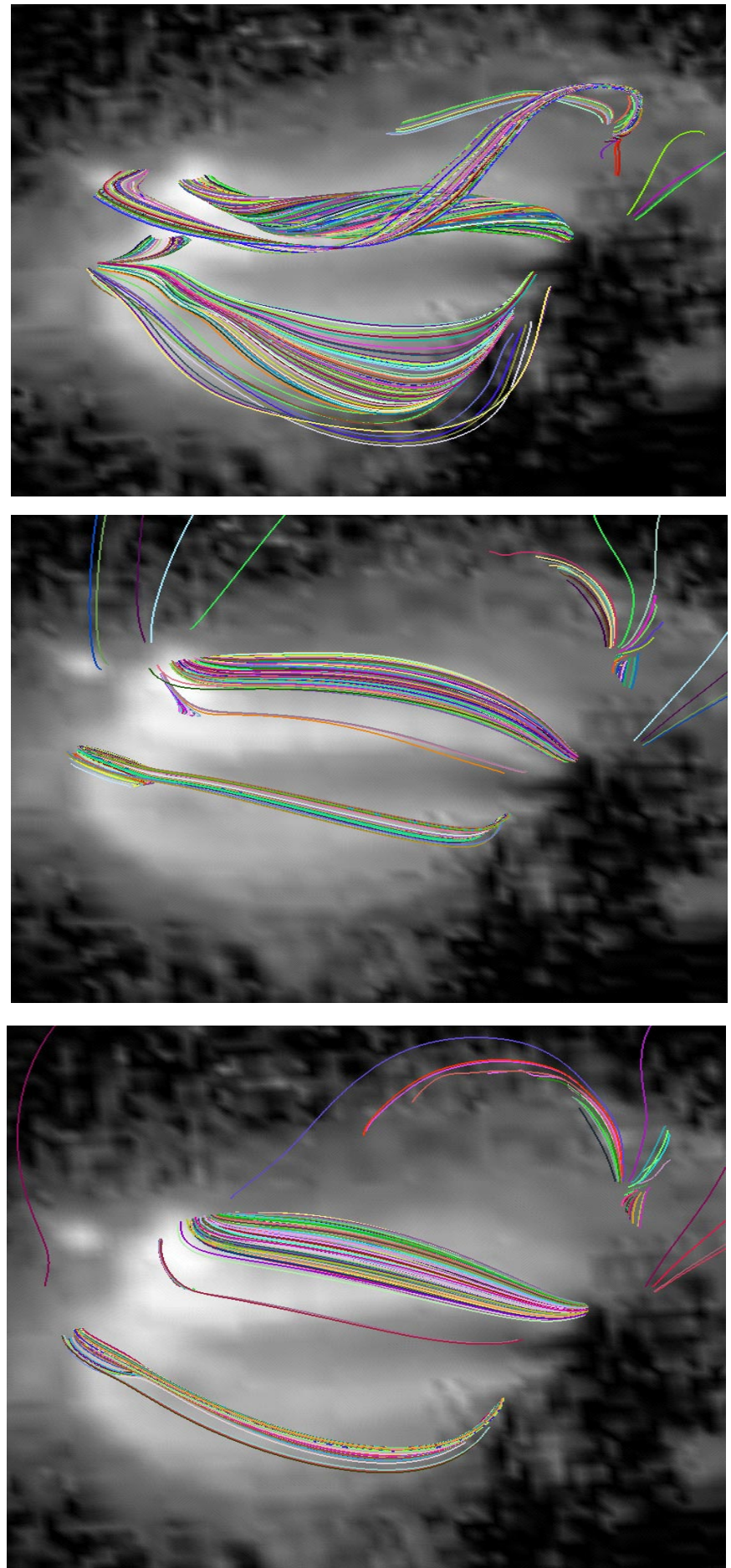

Fig. 8. Comparison of the overlay of the magnetic field lines for each type of reconstruction (non-linear (top), constant alpha (center), potential (bottom)) with soft X-ray (Yohkoh) image of the active region NOAA 7912 on 14th October 1995 at 00:20 UT.

issue since there is no method that ensures a $100 \%$ ambiguity resolution (Li 1994; Bleybel et al. 2002). Although confirming our results, those error estimates show that errors may be large for some vector magnetograms and therefore represent an important issue for force-free modelling, since errors on transverse fields are directly related to the current, which is the source of energy above the potential field. Therefore low-noise
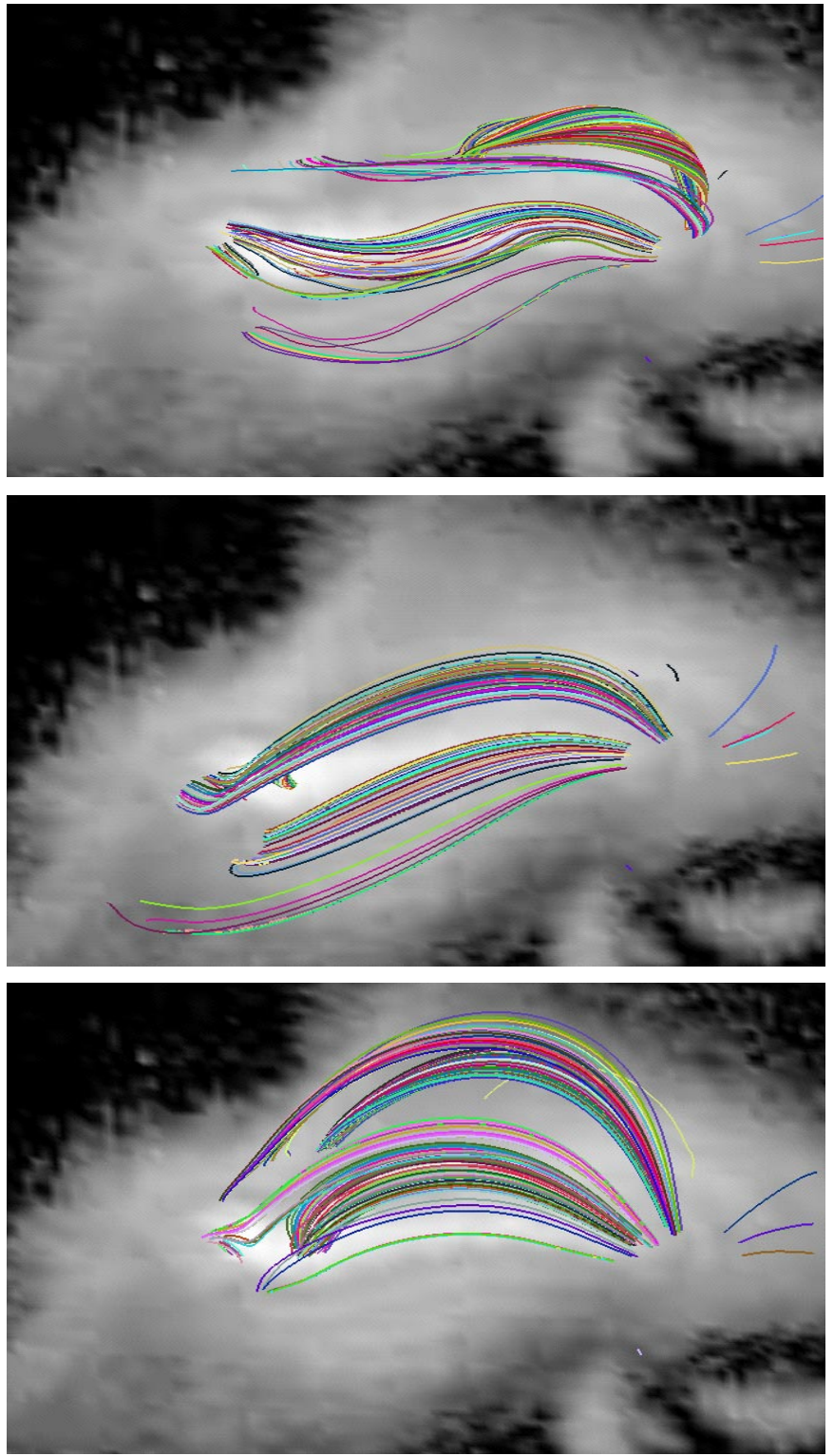

Fig. 9. Comparison of the overlay of the magnetic field lines for each type of reconstruction non-linear (top), linear having pre-flare helicity (center), linear with post-flare helicity (bottom) with soft X-ray (Yohkoh) image of the active region NOAA 7912 on 14th October 1995 at 17:55 UT.

measurements of the transverse field are more important than high resolution, which is crucial for further developement in the study of solar eruptions, as errors in most cases are too large to permit a viable analysis of physical phenomena responsible for the eruptive events. In our case, the large decrease in the energy between the pre-eruptive and post-eruptive states (about $31 \%$ of the mean value) has permitted us to reach conclusive results.

\section{Discussion}

Our results may be summarized as follows:

(i) The total magnetic energy decreased over the course of the eruption. 
(ii) The total relative helicity in the AR also decreased after the eruption and

(iii) The relaxed post-eruptive state is not consistent with a linear force-free state.

Point ii) suggests that helicity was ejected out of the active region. Coronal plasma has very little resistivity, and one may expect that it must then have a conserved global helicity. The fact that helicity decreased in the AR may only be explained by ejection of helicity out of the computational box. Recent numerical models and MHD simulations (Mikic \& Linker 1994; Amari et al. 1996) show this to be a possibility indeed; these models show the opening of the field over a finite time and the ejection of a plasmoid, which corresponds to a twisted flux rope. The final state in such models corresponds to a semi-open configuration, i.e. most of the field lines are open, though some of them remain closed. In our calculation, the ejected twisted flux tube corresponds to the magnetic cloud, which passes entirely out of the numerical box used for the reconstruction of the post-flare state. Note that mean $\alpha$ is positive. Note also that although mean $\alpha$ in the pre-eruptive state is smaller than the mean $\alpha$ in the post-eruptive state, the respective helicities are in reverse order, because $B_{z}$ is not exactly the same in the two states. Taylor (e.g. Taylor 1986) proposed that after a turbulent disruption, the magnetic field configuration relaxes to a linear i.e. constant $\alpha$ force-free state having the same helicity as the pre-disruptive state. Recall that the Taylor conjecture would apply for a constant helicity magnetic field configuration, even though the magnetic energy may change (e.g. due to reconnection). We have computed the linear force-free state having the same helicity as the pre-eruptive state. We found that this state (Fig. 9 - center) is not consistent with the full Taylor conjecture. However, since part of the helicity is ejected with the magnetic cloud (out of the computational box), the "remaining" helicity in the computational box might be used to fix the linear force-free state in a "weak version" of the Taylor conjecture. Our results show that this constant $\alpha$ state is not the state observed after the eruption (Fig. 9 - bottom).

There is an observed $13.48 \%$ decrease in unsigned flux between 00:20 and 17:55; we cannot attribute this decrease to the eruption (which occurred at about 05:00), since the active region may have lost the flux before the disruption; in fact, some studies have proposed that flux decrease can lead to disruption (Amari et al. 2000).

These inconsistencies with the Taylor conjecture in the astrophysical context have already been discovered in numerical MHD simulations of confined disruptions (Amari \& Luciani 1999). These authors proposed that helicity redistribution occurs in such systems either at the boundary of the domain (that is at infinity in the magnetic cloud, in the case of an unconfined disruptive event), or in current "sheet" non-linearities near the confining configuration (in the case of a confined disruptive event).

Some adequate constraints might be applied to prevent the relaxed state reaching the constant- $\alpha$ force-free field. Taylor's hypothesis results from a variational principle to obtain the minimum energy state in a configuration whose boundary is a magnetic surface, and may be generalized to a non-vanishing normal magnetic field component on the boundary with a relative helicity. A serious problem with the application of Taylor's hypothesis to the solar corona might lie with the anchoring of field lines at footpoints in the photosphere which may in turn inhibit the "ergodicity" in the magnetic configuration. In the absence of a rigid photosphere (closed configuration) this would lead to the state of constant $\alpha$ after full turbulent inverse cascades to larger scales, by a series a homogenizations of $\alpha$ in finite sub-domains. This may be difficult to achieve or unlikely to happen in the case of field lines having their footpoints anchored, and so the constant $\alpha$ state may well be unreachable. The main conclusion is that we proposed that this could be solved by finding a variational formulation in which the action functional would contain a term describing the fact that the field lines are rooted in the denser photosphere, in the same way that imposing helicity prevents the minimizer from otherwise being the current free state when $B_{z}$ is fixed. This formulation has not been found so far and is therefore an open problem.

Note, however, that since the total flux decreased $13.48 \%$ between the pre-eruptive and post-eruptive states, our study does not counter Taylor's conjecture with absolute certainty, although the percentage flux decrease is small compared to the helicity loss (about 50\%). Note that our analysis is validated by the estimations of errors in Table 1. It is worth clarifying that Taylor's hypothesis is generally invoked for the reconstruction problem as follows: one chooses $\alpha$ so as to fit loops in the soft $\mathrm{X}$-ray image of the active region. Usually, this value of $\alpha$ obtained afterward is different from the mean value of $\alpha$ in the active region, and they are both different from the value of $\alpha$ that gives the correct helicity (i.e. that is calculated using a nonlinear force-free model).

The success of the linear force-free model in some cases is simply a statement of the consistency of such a model with one particular structure corresponding to the particular length scale (loop) associated with this value of $\alpha$. Different values of $\alpha$ would be needed for each different structure. Therefore this is not exactly the Taylor conjecture that is used since the actual value of relative helicity is usually unknown. However it is interesting to note that these constant $\alpha$ models (which are very efficient in terms of CPU) may be successfully used to focus on a single isolated structure, and since these methods generally do not rely on the transverse magnetic field, one would be able to use the set of values of $\alpha$ corresponding to different structures contained in a magnetic configuration as boundary data for a consistent non-linear force-free computation, in order to access physical quantities and processes occurring in the configuration.

Finally it is worth noticing that the issue addressed here about the weaker version of Taylor's conjecture can only be solved by use of full time-dependent MHD simulations, taking the equilibrium preflare state as an initial state of the stability analysis. This is currently being investigated by the authors and will presented in a forthcoming paper.

Acknowledgements. This work has been supported by a NATO Collaborative Research Grant. The authors thank the MSSL SURF for Yohkoh/SXT data. LvDG is supported by Research Fellowship 
F/01/004 of the K.U. Leuven and by the Hungarian Government grants OTKA T032846 and T-038013.Data used here from Mees Solar Observatory, University of Hawaii, are produced with the support of NASA grant NAG 5-4941 and NASA contract NAS8-40801. We acknowledge constructive comments from an anonymous referee.

\section{References}

Aly, J. J. 1984, ApJ, 283, 349

Amari, T., Boulmezaoud, T. Z., \& Maday, Y. 1998, A\&A, 339, 252

Amari, T., \& Démoulin, P. 1992, in Proceedings of Workshop Méthodes de détermination des champs magnétiques solaires et stellaires, Observatoire de Paris, 187

Amari, T., Luciani, J. F., Aly, J. J., \& Tagger, M. 1996, ApJ, 466, L39 Amari, T., Luciani, J. F., \& Mikic, Z. 1999, Plasma Phys. and Control. Fusion, 41, 779

Amari, T., Aly, J. J., Luciani, J. F., Boulmezaoud, T. Z., \& Mikic, Z. 1997, Sol. Phys., 174, 129

Amari, T., Boulmezaoud, T. Z., \& Mikic, Z. 1999, A\&A, 350, 1051

Amari, T., \& Luciani, J.-F. 2000, Phys. Rev. Lett., 84, 1196

Amari, T., Luciani, J. F., Mikic, Z., \& Linker, J. 2000, ApJ, 529, L49

Antiochos, S. K., Devore, C. R., \& Klimchuk, J. A. 1999, ApJ, 510, 485

Antiochos, S. K., Klimchuk, J., \& Devore, R. C. 1999, ApJ, 466, L39

Baty, H. 2001, A\&A, 367, 321-325

Bhattacharjee, A., Dewar, R. L., \& Monticello, D. A. 1980, Phys. Rev. Lett., 45, 347

Bineau, M. 1972, Comm. Pure and Applied Math., 25, 77

Bleybel, A., Amari, T., \& Klimchuk, J. A. 2002, in preparation

Boulmezaoud, T. Z., \& Amari, T. 2000, Z. Angewandte Math. Phys., 51,942

Berger, M. A., \& Field, G. B. 1984, J. Fluid Mech., 35, 147

Burlaga, L. F., Sittler, E., Mariani, F., \& Schwenn, R. 1981, J. Geophys. Res., 86, 6673

Canfield, R. C., de La Beaujardiere, J.-F., Fan, Y., et al. 1993, ApJ, 411,362
Démoulin, P., Cuperman, S., \& Semel, M. 1992, A\&A, 263, 351

DeVore, R. C., \& Antiochos, S. K. 2000, ApJ, 539, 954

Finn, J. M., \& Antonsen, T. M. 1985, Comments Plasma Phys. Controlled Fusion, 26, 111

Heyvaerts, J., \& Priest, E. R. 1984, A\&A, 137, 63

Hood, A. W., \& Priest, E. R. 1979, Sol. Phys., 64, 303

Jefferies, J., Lites, B. W., \& Skumanich, A. 1989, ApJ, 343, 920

Klimchuk, J. A., Canfield, R. C., \& Rhoads, J. E. 1992, ApJ, 385, 327

Lepping, R. P., Burlaga, L. F., Szabo, A., et al. 1997, J. Geophys. Res., 102,14049

Li, J. 1994, Methods for the removal of the $180^{\circ}$ sign ambiguity in vector magnetograph, Ph.D. Thesis, Université Paris VII and Observatoire de Meudon, France

Linker, K., Lionello, R., Mikic, Z., \& Amari, T. 2001, J. Geophys. Res., 106, 25165

Linton, M. G., Longcope, D. W., \& Fisher, G. H. 1996, ApJ, 469, 954

López Fuentes, M. C., Démoulin, P., Mandrini, C. H., \& van DrielGesztelyi, L. 2000, ApJ, 544, 540

Low, B. C. 1994, Phys. Plasmas, 1, 1684

Mandrini, C. H., van Driel-Gesztelyi, L., Thompson, B., et al. 2000, Geofísica Internatcional, 39, 73

McClymont, A. N. 1997, Sol. Phys., 174, 191

Mickey, D. L., Canfield, R. C., Labonte, B. J., et al. 1996, Sol. Phys., 168,229

Mikic, Z., \& McClymont, A. N. 1994, in Solar Active Region Evolution - Comparing Models with Observtions, ed. K. S. Balasubramaniam, \& G. Simon, ASP Conf. Ser.

Pevtsov, A. A., Canfield, R. C., \& Metcalf, T. R. 1995, ApJ, 440, L109

Pevtsov, A. A., Canfield, R. C., \& McClymont A. N. 1997, ApJ, 481, 973

Raadu, M. A. 1972, Sol. Phys., 22, 425

Roumeliotis, G. 1997, ApJ, 481, 973

Sakurai, T. 1981, Sol. Phys., 69, 343

Taylor, J. B. 1986, Rev. Mod. Phys., 58, 741

van Driel-Gesztelyi, L., Manoharan, P. K., Démoulin, P., et al. 2000, J. Atm. Solar-Terr. Phys., 62/16, 1437 